\title{
Tarifpolitischer Jahresbericht 2013: Reale Tarifsteigerung und Anhebung von Niedriglöhnen
}

Die Tarifrunde 2013 knüpfte an die Entwicklung des Vorjahres an: Es gelang den Gewerkschaften, Tarifabschlüsse von durchschnittlich über $3 \%$ durchzusetzen. Die Abschlussraten fielen zwar etwas geringer aus als im Vorjahr, aber die jahresbezogene Tarifsteigerung führte angesichts der verhaltenen Entwicklung der Verbraucherpreise dennoch zu einem realen Anstieg der Tariflöhne und -gehälter von gut $1 \%$. Langwierige, zum Teil konfliktreiche, aber auch erfolgreiche Tarifauseinandersetzungen gab es in Niedriglohnbranchen, so zum Beispiel im Bewachungsgewerbe, im Friseurhandwerk, in der Leiharbeit und auch im Einzelhandel. Begleitet wurde die Tarifrunde in diesem Bereich von der politischen Diskussion um die Einführung eines allgemeinen gesetzlichen Mindestlohns. Die aktuelle Tarifrunde 2014 steht unter leicht verbesserten wirtschaftlichen Vorzeichen.

REINHARD BISPINCK, WSI-TARIFARCHIV

\section{Das Tarifjahr im Überblick}

\subsection{Rahmenbedingungen}

Die Vorbereitung jeder Tarifrunde wird maßgeblich von den wirtschaftlichen Rahmenbedingungen beeinflusst. Dabei spielen auch die (auf Prognosen gestützten) Erwartungshaltungen der Tarifparteien eine wichtige Rolle. Die gesamtwirtschaftlichen Rahmenbedingungen der Tarifrunde 2013 waren keineswegs rundum positiv: Bereits seit 2011 hatte sich das wirtschaftliche Wachstum kontinuierlich abgeschwächt. Im Jahr 2012 waren die realen Zuwachsraten des Bruttoinlandsprodukts (BIP) im Vorjahresvergleich von 1,8 \% im 1. Quartal über 0,6\% und $0,4 \%$ auf $0,0 \%$ im 4 . Quartal zurückgegangen. Wegen des BIP-Rückgangs im letzten Quartal 2012, im Vergleich zum Vorquartal um 0,5 \%, war sogar vorübergehend eine Rezessionsentwicklung in Deutschland nicht ausgeschlossen.

Für 2013 sahen die Konjunkturprognosen mehrheitlich nur ein schwaches Wachstum von unter einem Prozent vorher (z. B. IMK 2012). Die Entwicklung auf dem Arbeitsmarkt war 2012 und 2013 auf den ersten Blick stabil. Erwerbstätigkeit und sozialversicherungspflichtige Beschäftigung stiegen an, aber dies schlug nur teilweise auf die Arbeitslosigkeit durch. Saisonbereinigt stieg die Zahl der Arbeitslosen seit Beginn des Jahres 2012 kontinuierlich, wenn auch nur in geringem Umfang an (BA 2014).

Erstaunlicherweise blieb die rezessive wirtschaftliche Entwicklung in Europa, insbesondere in den südeuropäischen Krisenländern, für die deutsche Diskussion weitgehend ohne Bedeutung. Im offiziellen politischen Diskurs herrschte das Paradigma steigender Wettbewerbsfähigkeit durch „Strukturreformen“ am Arbeitsmarkt und Schuldenreduktion durch strikte Haushaltskonsolidierung vor (BMWi 2013). Auf europäischer Ebene wurde der Lohnpolitik in den Krisenländern vorrangig die Funktion der internen Abwertung zugedacht mit zum Teil hochproblematischen Folgen für die Tarifsysteme und die reale Lohnentwicklung (Schulten 2013; Schulten/Müller 2013). Mit Blick auf die deutsche Lohnpolitik wurde in der öffentlichen Diskussion überwiegend eingeräumt, dass eine Stabilisierung der privaten Nachfrage durch "angemessene“ Tarifabschlüsse sinnvoll sei. Während die Mainstream-Ökonomen einen lohnpolitischen Verteilungsspielraum von rund $2 \%$ sahen, plädierten keynesianische Ökonomen für einen Lohn-Zuschlag und sprachen sich für gesamtwirtschaftliche Lohnsteigerungen von 4 bis $5 \%$ aus (Bofinger 2013; Horn 2013). Diese stärkere Lohnsteigerung sollte die Binnennachfrage steigern und darüber auch die europäische Wirtschaft insgesamt stabilisieren. Eine stärkere Lohnentwicklung in Deutschland sei, so ein weiteres Argument, auch längerfristig zwingend erforderlich, um die großen 
Leistungsbilanzungleichgewichte im Euroraum schneller abzubauen (Herzog-Stein et al. 2013). In der lohn- und verteilungspolitischen Diskussion der Gewerkschaften spielten im Umfeld der Tarifrunde 2013 die wachsenden Probleme im Niedriglohnsektor und das Lohndumping in bestimmten Branchen ebenso eine Rolle wie die generelle Spreizung der Einkommens- und Vermögensverhältnisse (Brenke/Wagner 2013).

Die Lohn- und Gehaltsforderungen der Gewerkschaften bewegten sich zwischen 5 und 6,6\% und damit auf etwas niedrigerem Niveau als im Vorjahr (Bispinck/WSI-Tarifarchiv 2013a). Das wichtigste Signal kam zweifellos von der IG Metall. Mit ihrer Tarifforderung von 5,5\% für zwölf Monate für die Metallindustrie blieb sie einen Prozentpunkt unter ihrer Vorjahresforderung. Daraus konnte man entnehmen, dass ein Abschluss in Vorjahreshöhe nicht um jeden Preis angestrebt wurde. Die IG BAU lag mit ihrer Forderung von 6,6 \% für das Bauhauptgewerbe an der Spitze der größeren Tarifbranchen. In vielen Branchen wurden auch soziale Komponenten in Form von Mindesterhöhungen gefordert. In einzelnen Fällen wurden auch Tarifforderungen in Form von festen Eurobeträgen aufgestellt. Zwei Branchen fielen mit ihrem Forderungsvolumen aus dem Rahmen üblicher Lohnrunden. In beiden Fällen handelt es sich um ausgesprochene Niedriglohnbranchen: Im Gebäudereinigerhandwerk forderte die IG BAU eine Erhöhung der untersten Stundenlöhne in zwei Stufen um 70/65 ct (West) bzw. 98/98 ct (Ost), was einer Erhöhung um knapp 15 \% (West) bzw. knapp 26 \% (Ost) im Zeitraum von zwei Jahren entspricht. Im Bewachungsgewerbe Nordrhein-Westfalen ging ver.di noch weiter: Die Gewerkschaft forderte für die unterste Lohngruppe $(8,15 € /$ Std.) eine Erhöhung um 2,50 €, was einer Steigerung von rund $30 \%$ entspricht. Erklärtes Ziel war es, die durch geringe Einkommen geprägte Branche aus dem Niedriglohnsektor herauszuführen. Neben den reinen Entgeltforderungen spielten in einigen Tarifbereichen auch qualitative Tarifforderungen eine Rolle: Sie bezogen sich u. a. auf die Übernahme der Ausgebildeten, die Weiterentwicklung von Demografie-Tarifverträgen, die Fortschreibung von Altersteilzeitregelungen und die betriebliche Altersversorgung. Die Kündigungstermine in den einzelnen Branchen gaben folgenden zeitlichen Ablauf der Tarifrunde vor:

- Ende Dezember 2012 liefen die Tarifverträge für den öffentlichen Dienst (Länder), für die Deutsche Bahn AG, die Wohnungswirtschaft, die Holz und Kunststoff verarbeitende Industrie und einige Energiekonzerne aus.

- Im Februar 2013 endete die Laufzeit der Verträge in der Eisen- und Stahlindustrie und in weiteren Bereichen der Energiewirtschaft.

- Ende März folgten das Bauhauptgewerbe, das Versicherungsgewerbe und einige Bereiche des Einzelhandels und des Groß- und Außenhandels.

- Ende April standen die Verträge in der Metall- und Elektroindustrie, in der Kautschukindustrie, in weiteren Bereichen des Einzel- und Großhandels sowie des Kfz-Gewerbes zur Verhandlung an.
- Ende Mai folgten die Papierindustrie, das Textilreinigungsgewerbe, weitere Bereiche des Kfz-Gewerbes und Teile des ostdeutschen Einzelhandels.

- Im Juni stand u. a. das Volkswagenwerk auf dem Tarifkalender.

\subsection{Abschlüsse}

Wegen der lang laufenden Abschlüsse aus den Vorjahren gab es in einigen Branchen 2013 keine Lohnrunde. Das galt beispielsweise für den öffentlichen Dienst (Bund und Gemeinden), das Bankgewerbe, die chemische Industrie, die Deutsche Telekom AG und zahlreiche kleinere Branchen. Ein Blick auf die Tarifabschlüsse (Übersicht 1) zeigt folgendes Bild: ${ }^{1}$

Februar: Die ersten Abschlüsse erfolgten in verschiedenen Bereichen der Energiewirtschaft. So vereinbarten ver.di und IG BCE am 06.02. mit der Tarifgemeinschaft Energie (u. a. E.ON) eine Tarifsteigerung von $2,8 \% \mathrm{ab}$ 1.1.2013 und einer zusätzlichen Einmalzahlung von $300 €$ bei einer Gesamtlaufzeit von 13 Monaten.

März: Am 6.3. erreichte die IG Metall in der westdeutschen Eisen- und Stahlindustrie einen Abschluss mit einer Tarifsteigerung von 3,0 \% ab 1.3.2013 mit einer Laufzeit von 15 Monaten.

Im öffentlichen Dienst (Länder) erfolgte der Abschluss nach kräftigen Warnstreiks am 9.3. in der dritten Verhandlungsrunde: Er beinhaltet eine Tariferhöhung von 2,65 \% rückwirkend ab dem 1.1., eine Stufenerhöhung von 2,95 \% ab dem 1.1.2014 bei einer Laufzeit von insgesamt $24 \mathrm{Mo}$ naten.

Für die Deutsche Bahn AG vereinbarte die Eisenbahnund Verkehrsgewerkschaft (EVG) am 25.3. eine Pauschalzahlung von $500 €$ insgesamt für Januar bis April, eine Tarifanhebung von 3,0 \% ab 1.5.2013 und eine Stufenanhebung von 3,0 \% ab 1.4.2014 bei einer Gesamtlaufzeit von 19 Monaten.

April: Die IG BAU vereinbarte für das Bauhauptgewerbe am 5.4. in der dritten Verhandlungsrunde nach einem Nullmonat eine Tarifanhebung von 3,2 \% (West) und 4,0 \% (Ost) ab 1.5.2013 mit einer Laufzeit von 13 Monaten.

Am 7.4. wurde der heftige und lang anhaltende Tarifkonflikt im Bewachungsgewerbe NRW durch Schlichtung beigelegt. Ver.di konnte für die unterste Lohngruppe eine Anhebung der Tarifvergütung um 10,5 \% und für die Passagierkontrolle um 18,9\% (!) jeweils in einem Zeitraum von zwei Jahren durchsetzen. Dies stellt zweifelsohne den höchsten Abschluss der Tarifrunde 2013 dar.

Ebenfalls am 8.4. erfolgte der Abschluss im Hotel- und Gaststättengewerbe Bayern mit einer Pauschale von

1 Vgl. Einzelheiten zu den Tarifrunden in der Metallindustrie im öffentlichen Dienst (Länder) und Bewachungsgewerbe im Tarifpolitischen Halbjahresbericht 2013 (Bispinck/WSITarifarchiv 2013b) 
$45 €$ für den März und einer Tarifsteigerung von 4,0 \% ab 1.4.2013 bei einer Laufzeit von 17 Monaten.

Für die Deutsche Post $A G$ vereinbarte ver.di nach mehrtägigen Warnstreiks am 26.4. nach vier Nullmonaten (April bis Juli) eine Tarifanhebung von 3,1 \% ab 1.8.2013 sowie eine Stufenerhöhung von 2,6 \% ab 1.10.2014. Die Laufzeit beträgt 26 Monate. Die Tarifbeschäftigten sollen mindestens eine Tariferhöhung von $2.200 €$ bezogen auf die Laufzeit erhalten, ggf. erfolgt eine ergänzende Einmalzahlung.

Mai: In der Metall- und Elektroindustrie vereinbarte die IG Metall nach umfangreichen Warnstreiks im gesamten Bundesgebiet am 14.5. einen Pilotabschluss in Bayern, der nach zwei Nullmonaten eine Tarifsteigerung von 3,4 \% ab 1.7.2013 und eine Stufenanhebung von weiteren 2,2 \% ab 1.5.2014 umfasst. Die gesamte Laufzeit beträgt 20 Monate. Der Abschluss für die Volkswagen AG vom 28.5. hat ein vergleichbares Volumen und enthält zusätzlich einen Rentenbaustein von $300 €$.

Im Kfz-Gewerbe erfolgte der erste Abschluss am 22.5. ebenfalls für Bayern. Auch hier gab es zunächst zwei Nullmonate, anschließend ab 1.7. eine Tarifsteigerung von $2,8 \%$ und weitere $2,8 \%$ ab 1.8.2014 bei einer Laufzeit von 24 Monaten.

Juni: Für das Versicherungsgewerbe erreichte ver.di am 7.6. ein Tarifergebnis, das nach vier Nullmonaten eine Erhöhung der Tarifvergütungen um 3,2 \% ab 1.8.2013 und um weitere 2,2 \% ab 1.10.2014 vorsieht. Die Gesamtlaufzeit beträgt 24 Monate.

Im Groß- und Außenhandel beinhaltet der erste regionale Abschluss in Baden-Württemberg vom 14.6. nach zwei Nullmonaten eine Tarifanhebung um 3,0 \% ab 1.6.2013 sowie eine weitere Anhebung um 2,1 \% ab 1.4.2014 und eine zusätzliche Einmalzahlung.

Am 20.6. erreichte die IG BAU nach monatelangen Verhandlungen in der fünften Runde für das Gebäudereinigerhandwerk folgenden Abschluss: eine Tarifanhebung nach zwei Nullmonaten um 3,4/5,3 \% ab 1.1.2014 (West/Ost) und weitere 2,6/3,1 \% ab 1.1.2015. Das Tarifniveau Ost wird dadurch von 84 auf $86 \%$ angehoben. Bis spätestens Januar 2019 soll die Tarifangleichung vollständig realisiert sein.

September: Für die Leiharbeit-/Zeitarbeitsbranche erreichte die Tarifgemeinschaft des DGB nach sechs Verhandlungsrunden am 17.9. einen Abschluss, der in der untersten Entgeltgruppe einen Anstieg in drei Stufen von 8,19€ auf $9,00 € /$ Std. (West) bzw. von 7,50€ auf 8,50€/Std. (Ost) vorsieht. Die Laufzeit geht bis Ende 2016.

Dezember: Nach monatelangen Verhandlungen und Streiks konnte ver.di am 5.12.2013 für den Einzelhandel in Baden-Württemberg einen Pilotabschluss durchsetzen: Er sieht nach drei Nullmonaten eine rückwirkende Erhöhung der Tarifvergütungen ab dem 1.7. um 3,0 \% und eine weitere Erhöhung ab dem 1.4.2014 um 2,1 \% mit einer Laufzeit bis Ende März 2015 vor. Der arbeitgeberseitig gekündigte Manteltarifvertrag wurde wieder in Kraft gesetzt. Für sogenannte Verräumtätigkeiten wurde eine neue Vergütungsgruppe von 9,54/9,74 €/Std. ab 1.1./1.4.2014 vereinbart.

\section{Statistik der tariflichen Lohnent- wicklung}

Die DGB-Gewerkschaften schlossen 2013 in ganz Deutschland Lohn- und Gehaltstarifverträge für rund 13,1 Mio. Beschäftigte ab, davon für rund 11,1 Mio. in den alten und gut 2 Mio. in den neuen Bundesländern. Das entspricht knapp $67 \%$ der von Tarifverträgen erfassten Beschäftigten. Für weitere 4,3 Mio. Beschäftigte traten Stufenerhöhungen in Kraft, die bereits 2012 oder früher vereinbart wurden.

Bei rund 1,9 Mio. Beschäftigten liefen 2012 oder früher die Vergütungstarifverträge aus, aber es kam bis zum Jahresende (noch) nicht zu Neuabschlüssen bzw. laufende Tarifverträge sehen für 2013 keine Tarifanhebung vor.

\subsection{Abschlussrate}

Die tarifliche Abschlussrate beläuft sich gesamtwirtschaftlich im Durchschnitt auf 5,9 \% (2012: 5,0 \%), in Westdeutschland beträgt die Rate 5,5\%, in Ostdeutschland 8,2 \%. Die Abschlussrate schließt alle, ggf. auch 2014 und später in Kraft tretenden tabellenwirksamen Erhöhungen ein. Nicht berücksichtigt werden Pauschalzahlungen und zusätzliche Einmalzahlungen, die sich nicht dauerhaft in den Tariftabellen niederschlagen.

Diese Gesamtabschlussraten sind nur von begrenzter Aussagekraft, weil sie sich immer auf die gesamte, je nach Tarifbereich sehr unterschiedlich lange Laufzeit der Tarifabkommen beziehen. Berücksichtigt man lediglich die im Jahr 2013 abgeschlossenen und auch in Kraft getretenen Tariferhöhungen, ergibt sich eine Abschlussrate von 3,3 \% (West: 3,2 \%, Ost: 4,1\%). Differenziert man diese Größe nach Wirtschaftsbereichen, dann ergibt sich für 2013 eine Streuung zwischen 2,7 \% im Bereich Gebietskörperschaften, Sozialversicherung und 5,5 \% im Bereich Private Dienstleistungen, Organisationen ohne Erwerbszweck. Zu berücksichtigen ist stets, dass die Erhöhungen zu verschiedenen Zeitpunkten im Jahresverlauf wirksam wurden und damit auch die Auswirkungen auf die Tarifvergütungen des Jahres 2013 unterschiedlich waren. Im Jahr 2013 spielten „Nullmonate" bei den Tarifabschlüssen eine stärkere Rolle als noch im Vorjahr. Für rund 11,4 Mio. (2012: 6,1 Mio.), das entspricht rund $87 \%$ der von Neuabschlüssen begünstigten Beschäftigten, gab es Tarifabschlüsse mit verzögerter Anpassung der Lohn- und Gehaltserhöhungen. Die Zahl der Verzögerungsmonate lag mit durchschnittlich 2,4 über dem Vorjahr mit einem Wert von 1,8 Monaten.

Rund $64 \%$ der betroffenen Beschäftigten mussten einen Monat oder zwei Monate auf die reguläre Tariferhöhung warten, weitere $16 \%$ erhielten nach drei Monaten ihre erste Tarifsteigerung, für $7 \%$ dauerte es vier Monate und länger. Lediglich für 0,9 Mio. (7 \%) der betroffenen Beschäftigten vereinbarten die Gewerkschaften als Ausgleich Pauschalzahlungen, die durchschnittlich $87 €$ (West: $85 €$, Ost: $92 €)$ im Monat betrugen. 
Ausgewählte Tarifforderungen und -abschlüsse in der Tarifrunde 2013

\begin{tabular}{|c|c|c|c|c|}
\hline \multirow[t]{2}{*}{ Abschluss } & \multirow[t]{2}{*}{ Tarifbereich } & \multirow[t]{2}{*}{ Forderung } & \multicolumn{2}{|c|}{ Lohn, Gehalt, Entgelt } \\
\hline & & & 2013 & 2014 \\
\hline 31.01. & Landwirtschaft & $5,9 \%$ & $\begin{array}{l}\text { Bundesempfehlung Landwirtschaft } \\
230 € \text { Pauschale insg. für } 11 / 2012 \text { bis } 02 / 2013 \\
\mathbf{3 , 7} \% \text { ab 03/2013 }\end{array}$ & $2,8 \%$ ab $07 / 2014$ bis $06 / 2015$ \\
\hline 27.02. & $\begin{array}{l}\text { Holz- und Kunststoff } \\
\text { Westfalen-Lippe }\end{array}$ & $5,0 \%$ & $\begin{array}{l}2 \text { Nullmonate } \\
\mathbf{3 , 0} \% \text { ab } 03 / 2013 \text { bis } 04 / 2014\end{array}$ & \\
\hline 01.03. & Steinkohlenbergbau & Reallohnzuwachs & $\begin{array}{l}630 € \text { Pauschale insg. für } 01 \text { bis } 03 / 2013 \\
3,5 \% \text { ab } 04 / 2013 \text { bis } 12 / 2014\end{array}$ & \\
\hline 06.03 & $\begin{array}{l}\text { Eisen- und Stahlindustrie, Nie- } \\
\text { dersachsen und Bremen, NRW }\end{array}$ & $5,0 \%$ & $3,0 \%$ ab $03 / 2013$ bis $05 / 2014$ & \\
\hline 09.03 & $\begin{array}{l}\text { Öffentlicher Dienst Länder } \\
\text { (o. Hessen) }\end{array}$ & $\begin{array}{l}6,5 \% \\
\text { mit sozialer Komponente }\end{array}$ & $2,65 \%$ ab 01/2013 & $2,95 \%$ ab $01 / 2014$ bis $12 / 2014$ \\
\hline 11.03. & Energiewirtschaft Ost (AVEU) & $6,5 \%$ & $\begin{array}{l}550 € \text { Pauschale insg. für } 03 \text { bis } 04 / 2013 \\
2,8 \% \text { ab } 05 / 2013\end{array}$ & $\begin{array}{l}\mathbf{1 , 8} \% \text { ab } 05 / 2014 \text { bis } 04 / 2015 \\
275 € \text { zusätzliche Einmalzahlung }\end{array}$ \\
\hline 25.03 & Deutsche Bahn AG & $\begin{array}{l}6,5 \% \\
\text { mit sozialer Komponente }\end{array}$ & $\begin{array}{l}500 € \text { Pauschale insg. für } 01 \text { bis } 04 / 2013 \\
3,0 \% \text { ab } 05 / 2013\end{array}$ & $3,0 \%$ ab $04 / 2014$ bis $07 / 2014$ \\
\hline 05.04 & Bauhauptgewerbe & $6,6 \%$ & $\begin{array}{l}1 \text { Nullmonat } \\
\mathbf{3 , 2} \% \text { West, Berlin-West } \\
\mathbf{4 , 0} \% \text { Ost } \\
\text { jew. ab 05/2013 bis } 04 / 2014\end{array}$ & \\
\hline 07.04. & Bewachungsgewerbe NRW & rd. $30 \% *$ & $\begin{array}{l}\text { unterste Gruppe: } \\
\mathbf{5 , 8} \% \text { ab } 05 / 2013 \\
\text { Aviation (Passagierkontrolle): } \\
\mathbf{1 0 , 0} \% \text { ab } 05 / 2013\end{array}$ & $\begin{array}{l}\mathbf{4 , 4} \% \text { ab 01/2014 bis } 12 / 2014 \\
\mathbf{8 , 1} \% \text { ab 01/2014 bis } 12 / 2014\end{array}$ \\
\hline 08.04 & Hotels und Gaststätten Bayern & $120 €$ & $\begin{array}{l}45 € \text { Pauschale für } 03 / 2013 \\
\mathbf{4 , 0} \% \text { ab } 04 / 2013 \text { bis } 07 / 2014\end{array}$ & \\
\hline 08.04 & Priv. Transport u. Verkehr NRW & $\begin{array}{l}6,5 \% \\
\text { mind. } 125 €\end{array}$ & $\begin{array}{l}3 \text { Nullmonate } \\
2,8 \% \text { ab } 06 / 2013 \text { bis } 05 / 2014\end{array}$ & \\
\hline 10.04 & Kautschukindustrie & $5,8 \%$ & $2,1 \%$ ab $05 / 2013$ & $1,1 \%$ ab $01 / 2014$ bis $06 / 2014$ \\
\hline 19.04 & Textilindustrie Ost & $5,0 \%$ & $\begin{array}{l}60 € \text { Pauschale für } 04 / 2013 \\
3,0 \% \text { ab } 05 / 2013\end{array}$ & $2,6 \%$ ab $07 / 2014$ bis $03 / 2015$ \\
\hline 26.04 & Deutsche Post AG & $\begin{array}{l}6,0 \% \\
\text { mind. } 140 €\end{array}$ & $\begin{array}{l}4 \text { Nullmonate } \\
3,1 \% \text { ab } 08 / 2013\end{array}$ & $\begin{array}{l}\mathbf{2 , 6} \% \text { ab } 10 / 2014 \text { bis } 05 / 2015 \\
\text { (Mindesterhöhung von } 2.200 € \text { je AN bezogen } \\
\text { auf die Laufzeit) }\end{array}$ \\
\hline 14.05 & Metallindustrie & $5,5 \%$ & $\begin{array}{l}2 \text { Nullmonate } \\
3,4 \% \text { ab } 07 / 2013\end{array}$ & $2,2 \%$ ab $05 / 2014$ bis $12 / 2014$ \\
\hline 22.05 & Kfz-Gewerbe Bayern & $5,5 \%$ & $\begin{array}{l}2 \text { Nullmonate } \\
2,8 \% \text { ab } 07 / 2013\end{array}$ & $2,8 \%$ ab $08 / 2014$ bis $04 / 2015$ \\
\hline 28.05 & Volkswagen AG & $5,5 \%$ & $\begin{array}{l}2 \text { Nullmonate } \\
3,4 \% \text { ab } 09 / 2013 \\
275 € \text { Einmalzahlung bzw. } 300 € \text { Altersvorsor- } \\
\text { gebetrag }\end{array}$ & $2,2 \%$ ab $07 / 2014$ bis $02 / 2015$ \\
\hline 06.06 & Süßwarenindustrie NRW & $5,5 \%$ & $\begin{array}{l}1 \text { Nullmonat } \\
3,0 \% \text { ab } 06 / 2013\end{array}$ & $2,6 \%$ ab $06 / 2014$ bis $03 / 2015$ \\
\hline 07.06. & Versicherungsgewerbe & $\begin{array}{l}6,5 \% \\
\text { mind. } 160 €\end{array}$ & $\begin{array}{l}4 \text { Nullmonate } \\
3,2 \% \text { ab } 08 / 2013\end{array}$ & $2,2 \%$ ab $10 / 2014$ bis $03 / 2015$ \\
\hline 14.06 & $\begin{array}{l}\text { Groß- und Außenhandel } \\
\text { Baden-Württemberg }\end{array}$ & $\begin{array}{l}6,5 \% \\
\text { mind. } 140 €\end{array}$ & $\begin{array}{l}2 \text { Nullmonate } \\
\mathbf{3 , 0} \% \text { ab } 06 / 2013\end{array}$ & $\begin{array}{l}2,1 \% \text { ab } 04 / 2014 \text { bis } 03 / 2015 \\
90 € \text { Einmalzahlung in } 04 / 2014\end{array}$ \\
\hline 20.06 & Gebäudereinigerhandwerk, Arb. & $\begin{array}{l}\text { uG: } 2 \text { Stufen } \\
\text { W: } 70 / 65 \mathrm{Ct} \\
\text { O: } 98 / 98 \mathrm{Ct}\end{array}$ & 2 Nullmonate & $\begin{array}{l}\mathbf{3 , 4 / 5 , 3} \% \text { ab 01/2014 } \\
\mathbf{2 , 6 / 3 , 1} \% \text { ab 01/2015 jew. West/Ost, bis 10/2015 }\end{array}$ \\
\hline 21.06 & $\begin{array}{l}\text { Priv. Verkehrsgewerbe } \\
\text { (Speditionen und Logistik) } \\
\text { Berlin, Brandenburg }\end{array}$ & $6,0 \%$ & $\begin{array}{l}2 \text { Nullmonate } \\
2,8 \% \text { ab } 07 / 2013\end{array}$ & $2,4 \%$ ab $07 / 2014$ bis $07 / 2015$ \\
\hline 24.06 & Kfz-Gewerbe Thüringen & $\begin{array}{l}5,5 \% \\
\text { zzgl. Strukturkomponente }\end{array}$ & $\begin{array}{l}2 \text { Nullmonate } \\
2,8 \% \text { ab } 08 / 2013\end{array}$ & $2,8 \%$ ab $12 / 2014$ bis $04 / 2015$ \\
\hline 17.09. & $\begin{array}{l}\text { Zeitarbeit/ } \\
\text { Leiharbeit (BAP, iGZ) }\end{array}$ & $\begin{array}{l}\text { uG West: von } 8,19 \text { in Stufen auf } 9,00 € / \text { Std. } \\
\text { Ost/Westniveau: } 100 \%\end{array}$ & 2 Nullmonate & $\begin{array}{l}\text { uG West: von 8,19 auf } \mathbf{8 , 5 0 / 8 , 8 0 / 9 , 0 0 ~} € \text { je Std. } \\
\text { uG Ost inkl. Berlin: von 7,50 auf } \\
\mathbf{7 , 8 6 / 8 , 2 0 / 8 , 5 0 ~} \text { je Std. } \\
\text { ab 01/2014|04/2015|06/2016 } \\
\text { ansonsten: } \\
\mathbf{3 , 8 / 4 , 8} \% \text { (West/Ost inkl. Berlin) ab 01/2014 } \\
\mathbf{3 , 5 / 4 , 3} \% \text { ab 04/2015 } \\
\mathbf{2 , 3 / 3 , 7} \% \text { ab 06/2016 bis } 12 / 2016\end{array}$ \\
\hline 11.11. & $\begin{array}{l}\text { Energiewirtschaft NRW } \\
\text { (GWE-Bereich) }\end{array}$ & $5,5 \%$ & $\begin{array}{l}300 € \text { Pauschale für } 11 / 2013 \\
2,4 \% \text { ab } 12 / 2013\end{array}$ & $2,0 \%$ ab $10 / 2014$ bis $09 / 2015$ \\
\hline 05.12 & $\begin{array}{l}\text { Einzelhandel** } \\
\text { Baden-Württemberg }\end{array}$ & $\begin{array}{l}1 € / \text { Std. } \\
1.800 € \text { Mindesteinkommen }\end{array}$ & $\begin{array}{l}3 \text { Nullmonate } \\
3,0 \% \text { ab } 07 / 2013\end{array}$ & $2,1 \%$ ab $04 / 2014$ bis $03 / 2015$ \\
\hline
\end{tabular}

* Unterste Lohngruppe: $+2,50 €$ (bislang: 8,15€), Aviation (Passagierkontrolle): von $12,36 €$ auf $16,00 €$ je Std.

** Neue Lohngruppe für ab 01/2014 neu eingestellte AN mit ausschließlicher Warenverräum- und Auffülltätigkeit: 9,54 bzw. 9,74 € je Std. ab 01/2014 bzw. 04/2014. Quelle: WSI-Tarifarchiv; Stand: Dezember 2013. 
TABELLE 1

Laufzeit der Tarifverträge

Angaben in Monaten

\begin{tabular}{|c|c|c|c|c|c|c|c|c|c|c|c|c|}
\hline & 2002 & 2003 & 2004 & 2005 & 2006 & 2007 & 2008 & 2009 & 2010 & 2011 & 2012 & 2013 \\
\hline Gesamt & 18,3 & 20,5 & 21,8 & 25,7 & 22,1 & 22,2 & 22,4 & 24,1 & 24,3 & 22,8 & 18,0 & 22,8 \\
\hline West & 18,1 & 20,4 & 21,8 & 25,2 & 21,6 & 22,2 & 22,2 & 24,3 & 23,6 & 22,6 & 17,9 & 22,4 \\
\hline Ost & 19,7 & 21,0 & 22,0 & 28,4 & 24,7 & 21,9 & 23,4 & 23,2 & 28,3 & 23,9 & 19,1 & 24,7 \\
\hline
\end{tabular}

\subsection{Laufzeiten}

Der seit rund zehn Jahren zu beobachtende Trend zu fast zweijährigen Laufzeiten hat sich im vergangenen Jahr fortgesetzt. Die Laufzeit der Vergütungstarifverträge im Jahr 2013 beträgt durchschnittlich 22,8 Monate (2012: 18 Monate). Für rund 7 Mio. Beschäftigte (54,3\%) laufen die Abkommen zwischen 24 Monate und länger, für rund 3,6 Mio. (27,7 \%) betrug die Laufzeit genau 20 Monate. In den neuen Bundesländern laufen die neu abgeschlossenen Tarifverträge im Schnitt 2,3 Monate länger als in den alten (Tabelle 1).

\subsection{Jahresbezogene Tarifsteigerung}

Bei der Berechnung der auf das Kalenderjahr bezogenen Steigerung der tariflichen Grundlöhne und -gehälter werden im Unterschied zur tariflichen Abschlussrate die Auswirkungen aus der unterschiedlichen Lage und Laufzeit der Tarifabkommen berücksichtigt. Auch werden ggf. im Berichtsjahr wirksam werdende Abschlüsse aus den Vorjahren sowie zusätzliche Einmalzahlungen und Pauschalzahlungen als Ausgleich für Abschlussverzögerungen mit einbezogen. Die jahresbezogene Tarifsteigerung setzt die durchschnittliche tarifliche Grundvergütung des gesamten Jahres 2013 zum Vorjahr in Bezug und erfasst insgesamt 17,6 Mio. Arbeitnehmerinnen und Arbeitnehmer.

Diese kalenderjährliche Steigerung der Tarifverdienste 2013 (Tabelle 2) gegenüber 2012 beträgt für ganz Deutschland 2,7 \% (2012: 2,7 \%). Am höchsten fällt die jahresbezogene Tarifsteigerung mit 3,5 \% im Bereich Energie- und Wasserversorgung, Bergbau aus, gefolgt vom Baugewerbe mit 3,3\% und dem Investitionsgütergewerbe mit 3,0 \%. Im unteren Bereich rangieren die Gebietskörperschaften, Sozialversicherung mit 2,6\%, das Verbrauchsgütergewerbe mit 2,4\% und mit jeweils 2,3\% die Finanzdienstleistungen, der Handel sowie der Bereich Verkehr und Nachrichtenübermittlung. In Ostdeutschland liegt die kalenderjährliche Erhöhung mit 3,2 \% deutlich höher als in Westdeutschland mit 2,6 \%. Die Tarifsteigerung 2013, die sich für die länger laufenden
Abschlüsse aus dem Vorjahr ergibt, liegt mit 2,6 \% in etwa auf der Höhe der Neuabschlüsse mit 2,7 \%.

In Tarifbereichen mit rund einer Million Beschäftigten liefen Vergütungstarifverträge in den Jahren 2012 und 2013 aus, ohne dass neue Abschlüsse getätigt wurden. Bezieht man diese Bereiche mit ein, ergibt sich eine jahresbezogene Tarifsteigerung von 2,6\%. Die durchschnittliche jahresbezogene Tarifsteigerung 2013 von 2,7 \% liegt deutlich über dem Anstieg der Lebenshaltungskosten von $1,5 \%$. Real stiegen die tariflichen Grundvergütungen im gesamtwirtschaftlichen Durchschnitt um $1,2 \%$.

\subsection{Entwicklung der Effektivverdienste}

Die Effektivverdienste sind im vergangenen Jahr schwächer gestiegen als im Vorjahr. Die Summe der Bruttolöhne und -gehälter stieg 2013 um 3,1 \%. Je Beschäftigten ergibt sich auf Monatsbasis - nominal - ein Anstieg um 2,2 \%, auf Stundenbasis um 2,5\%. Daraus ergibt sich, dass die Bruttoverdienste 2013 erneut real (preisbereinigt) gestiegen sind, und zwar um 0,7 \% auf Monatsbasis bzw. 1,0\% auf Stundenbasis. Damit ist, anders als im Vorjahr, für 2013 wieder eine leichte negative Lohndrift zu konstatieren. Auf Monatsbasis gerechnet beläuft sie sich auf 0,5\%.

Der neutrale Verteilungsspielraum belief sich im vergangenen Jahr auf 1,3\%, darin sind der Anstieg der Verbraucherpreise $(+1,5 \%)$ und der Arbeitsproduktivität je Beschäftigten (-0,2\%), berücksichtigt. Berücksichtigt man dagegen die Stundenproduktivität $(+0,2 \%)$, ergibt sich ein Verteilungsspielraum von 1,5\%. Damit lag im vergangenen Jahr die tarifliche wie effektive Lohnentwicklung erkennbar über dem neutralen Verteilungsspielraum. Legt man dagegen als Maßstab die Zielinflationsrate der EZB (2\%) und die Trendproduktivität $(1,1 \%)$ zugrunde, bleibt die Lohnentwicklung auch 2013 unter dieser Marke.

Betrachtet man die funktionelle Einkommensverteilung, zeigt sich für das Jahr 2013 keine wesentliche Veränderung: Die Unternehmens- und Vermögenseinkommen stiegen um 2,8 \%, während die Arbeitnehmerentgelte um 2,9\% wuchsen. 


\subsection{Lohnangleichung Ost/West}

Die tarifliche Lohnangleichung zwischen Ost- und Westdeutschland hat keine Fortschritte gemacht. Für die tariflichen Grundvergütungen ergibt sich für den Stichtag 31.12.2013 folgendes Bild: Auf Basis von rund 50 Tarifbereichen/-branchen mit 1,7 Mio. erfassten Beschäftigten errechnet sich ein durchschnittliches Tarifniveau von $97,0 \%$. Gegenüber dem Vorjahr bleibt das Niveau demnach unverändert (Tabelle 3 ).

\subsection{Ausbildungsvergütungen}

Die Steigerung der tariflichen Ausbildungsvergütungen ist wie im vergangenen Jahr kräftiger ausgefallen als die allgemeine Tarifsteigerung. Nach Berechnungen des Bundesinstituts für Berufsbildung ergibt sich ein Anstieg von 4,2 \% (West: 4,1 \%, Ost: 5,0 \%) (BIBB 2014). Je nach Tarifbereich verbergen sich hinter diesen Durchschnittszahlen große Unterschiede: Gemessen an der Ausbildungsvergütung im dritten Ausbildungsjahr variiert die Steigerung in 26 ausgewählten Tarifbereichen zwischen 2,3 und 10,0 \%. In zwei Tarifbereichen sind die Ausbildungsvergütungen im vergangenen Jahr regional oder bundesweit gar nicht angehoben worden.

\section{Konflikte um Niedrig- und Mindest- löhne}

Im Vorfeld der Bundestagswahl spitzte sich die gesellschaftliche und politische Debatte um Niedriglöhne und ihre wirksame Bekämpfung zu. Die Parteien positionierten sich dabei mit unterschiedlichen Vorschlägen. SPD und Bündnis 90/Die Grünen forderten einen flächendeckenden einheitlichen Mindestlohn von 8,50 €/Std. Die Linke forderte einen Mindestlohn in Höhe von $10 € /$ Std. CDU und CSU sahen in ihrem Wahlprogramm vor, dass in Bereichen, in denen es keine Tarifverträge gibt, die Tarifpartner gemeinsam einen tariflichen Mindestlohn festlegen sollten. Weder nannte die Union einen Betrag, noch legte sie sich
TABELLE 2

Tarifsteigerung $2013^{1}$

Angaben in Prozent

\begin{tabular}{|c|c|c|c|}
\hline Wirtschaftsbereich & West & Ost & Gesamt \\
\hline Gartenbau, Land- und Forstwirtschaft & 2,5 & 3,1 & 2,7 \\
\hline Energie- und Wasserversorgung, Bergbau & 3,6 & 3,1 & 3,5 \\
\hline Grundstoff- und Produktionsgütergewerbe & 2,4 & 3,0 & 2,5 \\
\hline Investitionsgütergewerbe & 3,0 & 3,0 & 3,0 \\
\hline Verbrauchsgütergewerbe & 2,3 & 2,7 & 2,4 \\
\hline Nahrungs- und Genussmittelgewerbe & 2,9 & 3,0 & 2,9 \\
\hline Baugewerbe & 3,0 & 4,2 & 3,3 \\
\hline Handel & 2,3 & 2,2 & 2,3 \\
\hline Verkehr und Nachrichtenübermittlung & 2,3 & 2,7 & 2,3 \\
\hline Kreditinstitute, Versicherungsgewerbe & 2,3 & 2,3 & 2,3 \\
\hline Priv. Dienstleistungen, Organ. o. Erwerbszweck & 2,5 & 4,3 & 2,9 \\
\hline Gebietskörperschaften, Sozialversicherung & 2,6 & 2,6 & 2,6 \\
\hline Gesamte Wirtschaft & 2,6 & 3,2 & 2,7 \\
\hline
\end{tabular}

1 Jahresbezogene Erhöhung der tariflichen Grundvergütung 2013 gegenüber 2012

Quelle: WSI-Tarifarchiv; Stand: 31.12 .2013

auf einen einheitlichen Mindestlohn fest. Einzig die FDP lehnte einen Mindestlohn insgesamt ab. Das Thema Niedriglöhne spielte auch in der Tarifpolitik eine gewichtige Rolle. Die Gewerkschaften bemühten sich in einigen Branchen explizit um eine Anhebung der untersten Tarifvergütungen.

\subsection{Tarifverhandlungen}

Neben dem Bewachungsgewerbe und dem Gebäudereinigerhandwerk stand das Thema Niedrig- und Mindestlöhne u. a. in der Leiharbeitsbranche, im Friseurgewerbe und in der Fleischindustrie auf der Tagesordnung.

TABELLE 3

Tarifniveau Ost/West 2003 - 2013

Angaben in Prozent

\begin{tabular}{l|l|l|l|l|l|l|l|l|l|l|l|}
\hline Jahr & $\mathbf{2 0 0 3}$ & $\mathbf{2 0 0 4}$ & $\mathbf{2 0 0 5}$ & $\mathbf{2 0 0 6}$ & $\mathbf{2 0 0 7}$ & $\mathbf{2 0 0 8}$ & $\mathbf{2 0 0 9}$ & $\mathbf{2 0 1 0}$ & $\mathbf{2 0 1 1}$ & $\mathbf{2 0 1 2}$ & $\mathbf{2 0 1 3}$ \\
\hline$\%$ & 93,4 & 94,0 & 94,6 & 95,1 & 95,2 & 96,8 & 96,1 & 96,6 & 96,5 & 97,0 & 97,0 \\
\hline
\end{tabular}


TABELLE 4

Anhebung der tariflichen Entgeltgruppen in der Leih-/Zeitarbeit

Angaben in Euro/Std. und in Prozent

\begin{tabular}{ccccc}
\hline & \multicolumn{2}{c}{ unterste Gruppe (in $€ /$ Std.) } & \multicolumn{2}{c}{ übrige Gruppen (in \%) } \\
\hline & West & Ost inkl. Berlin & West & Ost inkI. Berlin \\
\hline 01.01 .2014 & 8,50 & 7,86 & 3,8 & 4,8 \\
\hline 01.04 .2015 & 8,80 & 8,20 & 3,5 & 4,3 \\
\hline 01.06 .2016 & 9,00 & 8,50 & 2,3 & 3,7 \\
\hline
\end{tabular}

Quelle: WSI-Tarifarchiv; Stand: 31.12.2013

TABELLE 5

Tariflicher Mindestlohn im Friseurgewerbe

Angaben in Euro/Std.

\begin{tabular}{lcc}
\hline & West & Ost, inkl. Berlin \\
\hline 01.08 .2013 & 7,50 & 6,50 \\
\hline 01.08 .2014 & 8,00 & 7,50 \\
\hline 01.08 .2015 & 8,50 & 8,50 \\
\hline
\end{tabular}

Quelle: WSI-Tarifarchiv; Stand: 31.12.2013.

Mitteilungen bisher, diese einzuklagen. Und schließlich konnten auch bei den Arbeitszeitkonten Verbesserungen für die Leiharbeitsbeschäftigten durchgesetzt werden (z. B. Vermeidung des Missbrauchs der Arbeitszeitkonten bei Teilzeitarbeit, Krankheit, betriebsbedingter Kündigung).

\subsubsection{Friseurgewerbe}

Das Friseurgewerbe gehört zu den traditionellen Niedriglohnbranchen. In seinem Niedriglohn-Monitoring stellte das WSI-Tarifarchiv für 2012 fest, dass 45 von 73 tariflichen Lohn- und Gehaltsgruppen in den regionalen Tarifverträgen, das entspricht $62 \%$, unterhalb der Grenze von 8,50€/ Std. angesiedelt sind. Ver.di gelang es im vergangenen Jahr mit einer Tarifgemeinschaft des Friseurhandwerks, an der 26 Tarifpartner beteiligt sind, ein Stufenmodell eines Mindestlohnes für in der Branche Beschäftigte zu vereinbaren (Tabelle 5). Für diesen Mindestlohn ist die Allgemeinverbindlicherklärung ab 1. November 2013 bereits erteilt.

Angesichts des sehr unterschiedlichen tariflichen Ausgangsniveaus bedeutete die Einführung des Mindestlohnes in den einzelnen Regionen entsprechend unterschiedliche Tarifanhebungen in der untersten Gruppe: Sie betrug im ersten Schritt (August 2013) 3,6 \% für die Angestellten im Tarifgebiet Rheinhessen, knapp $40 \%$ für die Angestellten in Berlin, über 73 \% für die Beschäftigten in Sachsen und etwas mehr als 113 \% für die Lohnempfänger in Brandenburg. Zu berücksichtigen ist, dass einige der bestehenden Tarifverträge schon seit zehn Jahren und länger nicht mehr angepasst wurden. In den meisten westdeutschen Tarifgebieten lag das unterste Tarifniveau im vergangenen Jahr bereits über 7,50€.

\subsubsection{Leiharbeit/Zeitarbeit}

Ende Oktober 2013 liefen die Tarifverträge der DGB-Tarifgemeinschaft mit den beiden Arbeitgeberverbänden der Zeitarbeit BAP und iGZ aus dem Jahre 2010 aus. Damals waren Tariferhöhungen in vier Schritten vereinbart worden, wonach die Mindestentgelte bis zum 1.11.2012 im Westen von 7,38 € auf 8,19 € pro Stunde, im Osten von $6,42 €$ im gleichen Zeitraum auf 7,50€ pro Stunde steigen sollten. Seit Mitte März 2013 führte die DGB-Tarifgemeinschaft Verhandlungen über die Erhöhung der Tarifvergütungen sowie weitere manteltarifliche Forderungen. ${ }^{2} \mathrm{Nach}$ sechs Verhandlungsrunden einigten sich die Tarifparteien auf die Anhebung der untersten Entgeltgruppe und eine entsprechende prozentuale Steigerung der übrigen Gruppen (Tabelle 4).

Weitere Ergebnisse: Der Einsatz von Leiharbeitsbeschäftigten als Streikbrecher in bestreikten Betrieben ist nicht mehr zulässig. Die missbrauchsanfälligen Entgeltgruppenbeschreibungen in den untersten Entgeltgruppen 1-4 konnten zugunsten der Beschäftigten geändert werden. Die verlängerten Ausschlussfristen in den Tarifverträgen haben zur Folge, dass die Leiharbeitsbeschäftigten bei Auseinandersetzungen um ihre Ansprüche aus den Tarifverträgen (z. B. bei Lohnstreitigkeiten) mehr Zeit haben als

\subsection{Tarifliche Mindestlöhne nach dem Ent- sendegesetz}

Auch im vergangenen Jahr waren die branchenbezogenen Mindestlöhne auf Basis des Arbeitnehmer-Entsendegesetzes Gegenstand von Verhandlungen und Anpassungen. Anfang vergangenen Jahres bestanden für elf Branchen gültige, allgemeinverbindliche Mindestlöhne. ${ }^{3}$ Hinzu kam die Lohnuntergrenze für die Leih-/Zeitarbeit auf Basis des Arbeitnehmerüberlassungsgesetzes (AÜG). Im Laufe des Jahres 2013 bis einschließlich Januar 2014 traten in zehn Branchen Er-

2 Die Verhandlungen als solche waren nicht unumstritten. In den Gewerkschaften gab es Forderungen, die Verträge Ende Oktober auslaufen zu lassen. Nach dem Arbeitnehmerüberlassungsgesetz (AÜG), so die Argumentation, hätte dann beim Fehlen von tariflichen Regelungen der Grundsatz Equal Pay gelten müssen (vgl. die Pro- und Contra-Argumente: DGB 2013a; verdi 2013; Labournet Dossier 2013).

3 In zwei Branchen, dem Gerüstbauerhandwerk sowie dem Steinmetz- und Steinbildhauerhandwerk, waren Anfang 2013 die bereits vereinbarten Mindestlöhne noch nicht für allgemeinverbindlich erklärt worden. 
ÜBERSICHT 2

Tarifliche Mindestlöhne nach EAntG - nur Branchen mit Steigerungen von 01/2013 bis 01/2014

Angaben in Euro und in Prozent

\begin{tabular}{|c|c|c|c|c|c|}
\hline \multirow[t]{2}{*}{ Branche } & \multirow[t]{2}{*}{ Beschäftigten-/Entgeltgruppe } & \multirow[t]{2}{*}{ Jan. 2013} & \multirow[t]{2}{*}{ Jan. 2014} & \multicolumn{2}{|c|}{ Steigerung } \\
\hline & & & & in $€$ & in $\%$ \\
\hline Abfallwirtschaft & Mindestlohn & $\begin{array}{c}8,33 \\
\text { (ausgelaufen 12/12) }\end{array}$ & 8,68 & 0,35 & 4,2 \\
\hline \multicolumn{6}{|l|}{ Bauhauptgewerbe } \\
\hline \multirow[t]{2}{*}{ West } & Werker (inkl. Berlin) & 11,05 & 11,10 & 0,05 & 0,5 \\
\hline & Fachwerker & 13,70 & 13,95 & 0,25 & 1,8 \\
\hline Berlin & Fachwerker & 13,55 & 13,80 & 0,25 & 1,8 \\
\hline Ost & Werker & 10,25 & 10,50 & 0,25 & 2,4 \\
\hline \multirow[t]{2}{*}{ Bergbau-Spezialgesellschaften } & Mindestlohn I & 11,53 & 11,92 & 0,39 & 3,4 \\
\hline & $\begin{array}{l}\text { Mindestlohn II } \\
\text { (Hauer/Facharbeiter) }\end{array}$ & 12,81 & 13,24 & 0,43 & 3,4 \\
\hline \multicolumn{6}{|l|}{$\begin{array}{l}\text { Berufliche Aus- und } \\
\text { Weiterbildung }\end{array}$} \\
\hline West inkl. Berlin & Pädagogische Mitarbeiter & 12,60 & 13,00 & 0,40 & 3,2 \\
\hline Ost & Pädagogische Mitarbeiter & 11,25 & 11,65 & 0,40 & 3,6 \\
\hline Dachdeckerhandwerk & Mindestlohn & 11,20 & 11,55 & 0,35 & 3,1 \\
\hline \multicolumn{6}{|l|}{ Elektrohandwerk (Montage) } \\
\hline West & Mindestentgelt & 9,90 & 10,00 & 0,10 & 1,0 \\
\hline Ost inkl. Berlin & Mindestentgelt & 8,85 & 9,10 & 0,25 & 2,8 \\
\hline \multicolumn{6}{|l|}{ Gebäudereinigerhandwerk } \\
\hline \multirow[t]{2}{*}{ West inkl. Berlin } & Innen- und Unterhaltsreinigung & 9,00 & 9,31 & 0,31 & 3,4 \\
\hline & Glas- und Fassadenreinigung & 11,33 & 12,33 & 1,00 & 8,8 \\
\hline \multirow[t]{2}{*}{ Ost } & Innen- und Unterhaltsreinigung & 7,56 & 7,96 & 0,40 & 5,3 \\
\hline & Glas- und Fassadenreinigung & 9,00 & 10,31 & 1,31 & 14,6 \\
\hline \multicolumn{6}{|l|}{ Maler- und Lackiererhandwerk } \\
\hline \multirow[t]{2}{*}{ West } & ungelernter Arbeitnehmer & 9,75 & 9,90 & 0,15 & 1,5 \\
\hline & Geselle & 12,00 & 12,15 & 0,15 & 1,3 \\
\hline Ost & Mindestlohn & 9,75 & 9,90 & 0,15 & 1,5 \\
\hline \multicolumn{6}{|l|}{ Pflegebranche } \\
\hline West inkl. Berlin & & 8,75 & 9,00 & 0,25 & 2,9 \\
\hline Ost & & 7,75 & 8,00 & 0,25 & 3,2 \\
\hline \multicolumn{6}{|l|}{$\begin{array}{l}\text { Steinmetz- und Steinbildhauer- } \\
\text { handwerk }\end{array}$} \\
\hline West inkl. Berlin & Mindestlohn & $11,00 * *$ & 11,00 & 0,00 & 0,0 \\
\hline Ost & Mindestlohn & $9,75 * *$ & 10,13 & 0,38 & 3,9 \\
\hline \multicolumn{6}{|l|}{$\begin{array}{l}\text { Wäschereidienstleistungen im } \\
\text { Objektkundengeschäft }\end{array}$} \\
\hline West & Mindestlohn & 8,00 & $8,25 *$ & 0,25 & 3,1 \\
\hline Ost inkl. Berlin & Mindestlohn & 7,00 & $7,50 *$ & 0,50 & 7,1 \\
\hline Zeit-/Leiharbeit & Lohnuntergrenze & & & & \\
\hline West & & 8,19 & $8,50 *$ & 0,31 & 3,8 \\
\hline Ost inkl. Berlin & & 7,50 & $7,86 *$ & 0,36 & 4,8 \\
\hline
\end{tabular}

* AVE noch nciht erteilt.

** AVE erstmal ab 10/13

Quelle: WSI-Tarifarchiv; Stand 31.12.2013

höhungen der Mindestlöhne zwischen 0,5 und 14,6\% in Kraft (Übersicht 2). Erstmalig wurde für das Steinmetz- und Steinbildhauerhandwerk ein tariflicher Mindestlohn in Höhe von $11 € /$ Std. bzw. 9,75€/Std. für West- bzw. Ostdeutschland festgesetzt, der zum 1.10.2013 in Kraft trat. Anfang 2014 bestanden damit in 14 Branchen tarifliche Mindestlöhne auf der Basis des Arbeitnehmerentsendegesetzes (AEntG) bzw. des Arbeitnehmerüberlassungsgesetzes (AÜG). In zwei dieser
Branchen (Wäschereidienstleistungen, Leih-/Zeitarbeit) sind die bereits vereinbarten Mindestlöhne zum Zeitpunkt des Redaktionsschlusses noch nicht allgemeinverbindlich erklärt worden. Der Tarifbereich Forstliche Dienstleister, für den bereits ein Mindestlohn ausgehandelt wurde, ist noch nicht im Geltungsbereich des AEntG enthalten. Der Mindestlohn für das Wach- und Sicherheitsgewerbe lief Ende 2013 aus. Eine neue Tarifvereinbarung gibt es noch nicht. 


\section{ÜBERSICHT 3}

\section{Vereinbarte Tarifanhebungen für 2014}

Angaben in Prozent

\begin{tabular}{|c|c|c|c|}
\hline Tarifbereich & $\%$ & ab ... 2014 & bis: \\
\hline Deutsche Bahn AG & 3,0 & 04 & $07 / 2014$ \\
\hline Deutsche Post AG & 2,6 & 10 & $05 / 2015$ \\
\hline Einzelhandel NRW & 2,1 & 05 & $04 / 2015$ \\
\hline Energiewirtschaft NRW & 2,0 & 10 & $09 / 2015$ \\
\hline Gebäudereinigerhandwerk West, Arb. & 3,4 & 01 & $12 / 2014^{*}$ \\
\hline Gebäudereinigerhandwerk Ost, Arb. & 5,3 & 01 & $12 / 2014^{*}$ \\
\hline Groß- und Außenhandel Baden-Württ. & 2,1 & 04 & $03 / 2015$ \\
\hline Kautschukindustrie & 1,1 & 01 & $06 / 2014$ \\
\hline Landwirtschaft & 2,8 & 07 & $06 / 2015$ \\
\hline Metallindustrie & 2,2 & 05 & $12 / 2014$ \\
\hline Öffentl. Dienst Länder o. Hessen u. Berlin & 2,95 & 01 & $12 / 2014$ \\
\hline Süßwarenindustrie NRW & 2,6 & 06 & $03 / 2015$ \\
\hline Textil-Bekleidung Ost & 2,6 & 07 & $03 / 2015$ \\
\hline Versicherungsgewerbe & 2,2 & 10 & $03 / 2015$ \\
\hline
\end{tabular}

* Weitere Anhebung um 2,6/3,1 \% (West/Ost) ab 01/2015 bis 10/2015 Quelle:WSI-Tarifarchiv; Stand: 31.12. 2013 geltende Mindestlohnniveau bis spätestens zum 1. Januar 2017 erreicht wird, durch Aufnahme in das Arbeitnehmerentsendegesetz.

(4) Regelmäßige Überprüfung des Mindestlohnes erstmals zum 10.6.2017 mit Wirkung zum 1. Januar 2018 - von einer Mindestlohnkommission der Tarifpartner, ggf. Anpassung und Erstreckung durch eine Rechtsverordnung.

(5) Benennung der Mitglieder der Kommission durch die Spitzenorganisationen der Arbeitgeber und Arbeitnehmer (Größe: 3 zu 3 plus Vorsitz); alternierender Vorsitz der Kommission; Hinzuziehung wissenschaftlichen Sachverstandes (ohne Stimmrecht) auf Vorschlag der Spitzenorganisationen (1 plus 1).

Diese Vereinbarung traf auf ein unterschiedliches Echo: Die Arbeitgeber qualifizierten den flächendeckenden gesetzlichen Mindestlohn als „Eingriff in die Tarifautonomie“ und verlangten, „wenn überhaupt, dann müssen Arbeitgeber und Gewerkschaften Lohnuntergrenzen miteinander vereinbaren“" (BDA 2013, S. 36). Die Gewerkschaften begrüßten dagegen die Umsetzung einer langjährigen Forderung (DGB 2013b). Unmittelbar nach Abschluss der Koalitionsvereinbarung setzte eine lebhafte Debatte über die Ausgestaltung und Umsetzung dieser Maßnahmen ein: Vertreter von CDU und insbesondere CSU sowie der Wirtschafts- und Arbeitgeberverbände setzten sich für weitreichende Ausnahmen beim Mindestlohn ein. Genannt wurden folgende Branchen und Personengruppen, die nicht vom Mindestlohn erfasst werden sollten: Landwirtschaft (insb. Saisonarbeiter), Briefzusteller, Taxifahrer, Hilfsarbeiter, Minijobber, Schüler, Praktikanten, Rentner, Langzeitarbeitslose, ehrenamtlich Tätige. Diese Forderungen stießen postwendend auf Ablehnung seitens der Gewerkschaften. Die neue Bundesarbeitsministerin Andrea Nahles machte deutlich, dass sie grundsätzlich keine Ausnahmen ins Gesetz aufnehmen wolle.

Ein Gutachten des wissenschaftlichen Dienstes des Deutschen Bundestages bezweifelte die Rechtmäßigkeit solcher Regelungen aus verfassungsrechtlicher Sicht (Bug 2014). Analysen auf Basis aktueller Einkommensdaten kamen zu dem Ergebnis, dass bis zu einem Drittel der Niedriglohn-Bezieher unter 8,50€ von einem Mindestlohn ausgeschlossen blieben, sollten die Ausnahmen umgesetzt werden (Amlinger et al. 2014).

Weitere Diskussionspunkte betrafen die Zusammensetzung und Aufgabenstellung der Mindestlohnkommission sowie den Zeitpunkt der Einführung und die Anpassung des Mindestlohnes.

4 Vgl. „Deutschlands Zukunft gestalten” Koalitionsvertrag zwischen CDU, CSU und SPD, 18. Legislaturperiode, Dezember 2013 


\section{Ausblick auf die Tarifrunde 2014}

Die ökonomischen Rahmenbedingungen der Tarifrunde 2014 sind etwas günstiger als im Vorjahr. Die Institute prognostizieren mehrheitlich ein reales Wachstum zwischen 1,2 und 2,0 \%. Das Institut für Makroökonomie und Konjunkturforschung (IMK) spricht immerhin von einer „Aufhellung der wirtschaftlichen Lage“. Nach seiner Prognose wird auf dem Arbeitsmarkt zwar die Zahl der Erwerbstätigen voraussichtlich leicht steigen, aber aufgrund von zunehmender Erwerbsneigung und Zuwanderung wird die Arbeitslosigkeit nicht zurückgehen (IMK 2013).

Auch in der Tarifrunde 2014 stehen Forderungen nach deutlichen Steigerungen der Tarifentgelte im Mittelpunkt. Die bislang bekannten Tarifforderungen bewegen sich zwischen 5,5 und 6,0 \%. Nebenforderungen zielen auf die Situation der Auszubildenden und Regelungen zum Altersübergang (Demografietarifverträge). Nach dem Kündigungsterminkalender werden im ersten Halbjahr 2014 folgende größere Tarifbereiche das Tarifgeschehen bestimmen: Die chemische Industrie, in der die Verträge zwischen Ende Dezember 2013 und Ende Februar 2014 ausliefen bzw. auslaufen. Im Februar folgt der öffentliche Dienst (Bund und Gemeinden), im April das Bauhauptgewerbe und das Bankgewerbe und im Mai die Eisen- und Stahlindustrie.

Seit mehr als zehn Jahren hat sich die Praxis eingebürgert, Vergütungstarifverträge mit längeren Laufzeiten zu vereinbaren. Dies hat zur Konsequenz, dass in den jährlichen Tarifrunden längst nicht mehr alle Branchen auch tatsächlich verhandeln. Im vergangenen Jahr wurden in einer Reihe von Tarifbereichen bereits Tariferhöhungen für das Jahr 2014 und teilweise auch für 2015 vereinbart, wie Übersicht 3 zeigt. Dazu zählen von den größeren Bereichen die Metall- und Elektroindustrie, der Einzelhandel und der öffentliche Dienst (Länder).

Die vorliegenden Abschlussraten für 2014 bewegen sich von Ausnahmen abgesehen zwischen 2,0 und 3,0 \% Die Erfahrung zeigt allerdings, dass diese tariflichen Steigerungsraten nur begrenzten Einfluss auf die anlaufenden Tarifrunden haben. Stufenanhebungen fallen in aller Regel niedriger aus als die Tarifsteigerungen in der ersten Phase eines mehrteiligen Tarifabschlusses.
Brenke, K./Wagner, G. (2013): Ungleiche Verteilung der Einkommen bremst das Wirtschaftswachstum, in: Wirtschaftsdienst 93 (2), S. 110-116 Bofinger, P. (2013): Wirtschaftsweiser fordert Lohnplus von fünf Prozent, http://www.spiegel.de/wirtschaft/unternehmen/peter-bofinger-wirtschaftsweiser-fordert-lohnplus-von-fuenf-prozent-a-875948.html)

Bug, A. (2014): Ausnahmen von einem gesetzlichen Mindestlohn für einzelne Arbeitnehmergruppen aus verfassungsrechtlicher Sicht. Wissenschaftliche Dienste des Deutschen Bundestages 6-3000-002/14

Bundesagentur für Arbeit (BA) (2014): Der Arbeits- und Ausbildungsmarkt in Deutschland, Monatsbericht Dezember und Jahr 2013, Nürnberg Bundesministerium für Wirtschaft und Technologie (BMWi) (2013): Jahres wirtschaftsbericht 2013: Wettbewerbsfähigkeit - Schlüssel für Wachstum und Beschäftigung in Deutschland und Europa, Berlin

Deutscher Gewerkschaftsbund (DGB) (2013a): Tarifverhandlungen Leiharbeit: Was muss ich wissen?, 6.5., Berlin, http://www.dgb.de/

themen/++co++8e936006-b66e-11e2-83f0-00188b4dc422

Deutscher Gewerkschaftsbund (DGB) (2013b): Bewertung der Koalitionsvereinbarung, 27.11., Berlin, http://www.dgb.de/repository/public_storage/ c5c7f838-56a6-11e3-8a9d-00188b4dc422/file/Bewertung-der-Koalitionsvereinbarungen.pdf

http://www.dgb.de/themen/++co++8e936006-b66e-11e2-83fo-

00188b4dc422?tab=Alle\&display_page=3\&k:list=Arbeit\&k:list=Leiharbeit/ Zeitarbeit

Herzog-Stein, A./Joebges, H./Stein, U./Zwiener, R. (2013): Arbeitskostenentwicklung und internationale Wettbewerbsfähigkeit in Europa - Arbeitsund Lohnstückkosten in 2012 und im 1. Halbjahr 2013, IMK Report (88), Dezember

Horn, G. (2013): „Euro-Krise: Forschungsinstitut fordert kräftige Lohnsteigerung", Spiegel Online 7.1., http://www.spiegel.de/wirtschaft/soziales/imkforscher-fordern-hoehere-loehne-a-876186.html

Institut für Makroökonomie und Konjunkturforschung (IMK), Arbeitskreis Konjunktur (2012): Auf des Messers Schneide - Prognose der wirtschaftlichen Entwicklung 2013, IMK Report (78), Dezember

Institut für Makroökonomie und Konjunkturforschung (IMK), Arbeitskreis Konjunktur (2013): Nur zögerliche Konjunkturbelebung. Prognose-Update: Deutsche Konjunktur zur Jahreswende 2013/2014, IMK-Report (89), Dezember

Labournet (2013): Dossier Leiharbeit, http://www.labournet.de/politik/alltag/ leiharbeit/tarifverhandlungen/in-aller-stille-tarifverhandlungen-zur-leiharbeit/ Schulten, Th. (2013): Europäischer Tarifbericht des WSI 2012/2013, in: WSIMitteilungen 66 (8), 588-597, http://www.boeckler.de/wsimit_2013_08_schulten.pdf

Schulten, Th./Müller, T. (2013): Ein neuer europäischer Interventionismus? Die Auswirkungen des neuen Systems der europäischen Economic Governance auf Löhne und Tarifpolitik, in: Wirtschaft und Gesellschaft 39 (3), $291-322$

ver.di Bundesvorstand (2013): Leiharbeit braucht Equal Pay und einen Tarifvertrag!, 27. Mai

\section{AUTOREN}

REINHARD BISPINCK, Dr., ist Abteilungsleiter des Wirtschafts- und Sozialwissenschaftlichen Instituts (WSI) und Leiter des WSI-Tarifarchivs in der HansBöckler-Stiftung.

reinhard-bispinck@boeckler.de

GÖTZ BAUER, MERLE FÖHR, ULRICH SCHMIDT, MONIKA SCHWACKE-PILGER, KATHRIN SONNEN, ANDREA TAUBE und MONIKA WIEBEL sind Sachbearbeiterinnen und Sachbearbeiter im WSI-Tarifarchiv.
Bispinck, R./WSI-Tarifarchiv (2013b): Tarifpolitischer Halbjahresbericht: Eine Zwischenbilanz der Lohn- und Gehaltsrunde 2013, in: WSI-Mitteilungen 66 (6), S. 423-434, http://www.boeckler.de/wsimit_2013_06_bispinck.pdf 